\title{
Sliding Mode Extremum Seeking Control Scheme Based on PSO for Maximum Power Point Tracking in Photovoltaic Systems
}

\author{
Her-Terng Yau, ${ }^{1}$ Chih-Jer Lin, ${ }^{2}$ and Chen-Han Wu ${ }^{1}$ \\ ${ }^{1}$ Department of Electrical Engineering, National Chin-Yi University of Technology, Taichung 41170, Taiwan \\ ${ }^{2}$ Graduate Institute of Automation and Technology, National Taipei University of Technology, Taipei 10608, Taiwan \\ Correspondence should be addressed to Her-Terng Yau; pan1012@ms52.hinet.net
}

Received 22 January 2013; Accepted 21 March 2013

Academic Editor: Fahrettin Yakuphanoglu

Copyright (c) 2013 Her-Terng Yau et al. This is an open access article distributed under the Creative Commons Attribution License, which permits unrestricted use, distribution, and reproduction in any medium, provided the original work is properly cited.

\begin{abstract}
An extremum seeking control (ESC) scheme is proposed for maximum power point tracking (MPPT) in photovoltaic power generation systems. The robustness of the proposed scheme toward irradiance changes is enhanced by implementing the ESC scheme using a sliding mode control (SMC) law. In the proposed approach, the chattering phenomenon caused by high frequency switching is suppressed by means of a sliding layer concept. Moreover, in implementing the proposed controller, the optimal value of the gain constant $\left(U_{0}\right)$ is determined using a particle swarm optimization (PSO) algorithm. The experimental and simulation results show that the proposed PSO-based sliding mode ESC (SMESC) control scheme yields a better transient response, steadystate stability, and robustness than traditional MPPT schemes based on gradient detection methods.
\end{abstract}

\section{Introduction}

With the gradual depletion of the world's supply of natural resources and mounting concern regarding the greenhouse effect and global warming, the need has emerged for new energy sources capable of meeting the world's power needs in a sustainable and environmentally friendly manner. In response to this requirement, many green energy sources have been proposed in recent decades, including photovoltaic power generation systems, wind farms, hydraulic power generation systems, fuel cells, and biomass energy generation systems. Among these various energy sources, photovoltaic power generation systems are particularly attractive since they have a simple architecture, high reliability, and an easily extendable generating capacity. However, the performance of photovoltaic modules is highly sensitive to the angle of the incident sunlight, the atmospheric temperature, the solar panel conversion efficiency, and so on. Therefore, to maximize the system efficiency and robustness toward environmental perturbations and changes in the irradiance conditions, effective control schemes are required $[1$, 2].
The literature contains many proposals for maximum power point tracking (MPPT) schemes in photovoltaic systems. For example, Femia et al. [3] proposed a perturbation and observation $(\mathrm{P} \& \mathrm{O})$ method in which the output voltage of each photovoltaic module was adjusted by increasing or reducing the load in accordance with observations of the output power before and after loading, respectively. However, in the proposed approach, the point of maximum power is reached via a process of successive adjustment, and thus poor stability, energy loss, and a low generating efficiency inevitably occur. Abdelsalam et al. [4] proposed an improved $\mathrm{P} \& \mathrm{O}$ scheme for MPP tracking in photovoltaic systems and showed that the proposed approach reduced oscillations during the tracking process and improved both the efficiency and the reliability of the overall power grid. Li and Wang [5] proposed an incremental conductance (IC) method in which the output voltage of each solar cell was increased or decreased depending on whether the output power of the cell was located to the left or the right of the MPP $(d P / d V=$ $0)$, respectively. However, the performance of the proposed method is dependent on a highly precise measurement of the output power, which is not easily achieved in practical 
implementations. Safari and Mekhilef [6] presented an improved IC method for solving the MPPT problem based on a direct control scheme. The experimental results showed that the proposed method enabled the MPP to be tracked rapidly and without excessive oscillation. Liu et al. [7] proposed an incremental MPPT algorithm in which the step size was adjusted automatically in accordance with changes in the voltage output of the photovoltaic modules. The experimental results showed that the proposed method improved both the speed and the accuracy of the MPPT process compared to a conventional fixed step size method. However, the method has poor robustness toward sudden changes in the irradiance conditions and leads to significant energy losses as a result. Koutroulis et al. [8] presented an MPPT control system consisting of a buck-type DC-DC converter regulated by a microcontroller. In the proposed approach, the DCDC converter was adjusted directly in accordance with the output power of the photovoltaic array, thereby reducing the complexity of the system. However, the efficiency of the proposed approach is highly sensitive to the measurement accuracy of the power signal. The authors in [9-11] proposed various extremum seeking control (ESC) techniques for solving the MPPT problem in photovoltaic systems. Compared to conventional adaptive control methods, which process only a given or present reference trajectory, ESC methods require no modeling and result in a more precise control performance. The output characteristic curve of a photovoltaic power generation system has a nonlinear form. Thus, conventional MPPT algorithms commonly converge toward a local MPP rather than the global MPP. It was shown in $[12,13]$ that ESC schemes provide an effective means of resolving this problem, thereby minimizing energy losses and ensuring the stability and robustness of the system.

Brunton et al. [13] presented a novel ESC algorithm based on a natural inverter ripple for MPP tracking in solar power systems. The experimental results showed that the proposed system was capable of tracking the MPP with an accuracy of more than $99 \%$ within a very short time. However, the proposed method has poor robustness and therefore suffers a severe performance loss given rapid changes in the irradiance conditions.

The present study proposes an enhanced ESC control scheme for solving the MPPT problem in photovoltaic systems. In the proposed approach, the robustness of the MPPT algorithm toward irradiance changes is enhanced by means of a sliding mode control (SMC) law [14]. Furthermore, the chattering phenomenon observed in traditional MPPT schemes is suppressed by means of a sliding layer concept. Finally, the response time and stability of the proposed controller are enhanced by using a particle swarm optimization (PSO) algorithm to determine the optimal value of the gain constant $\left(U_{0}\right)$. It is shown that the proposed method outperforms traditional MPPT schemes based on gradient detection methods in terms of a better transient response, an improved steady-state stability, and an improved robustness.

The remainder of this paper is organized as follows. Section 2 describes the application of the ESC technique to the MPPT problem and introduces the Sliding Mode ESC (SMESC) scheme proposed in the present study. Section 3

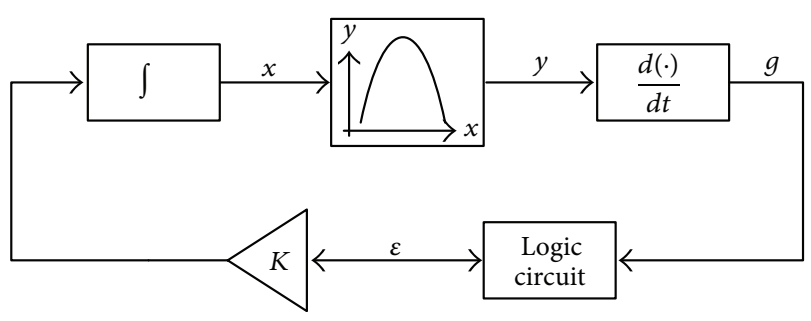

FIGURE 1: Block diagram of general ESC scheme.

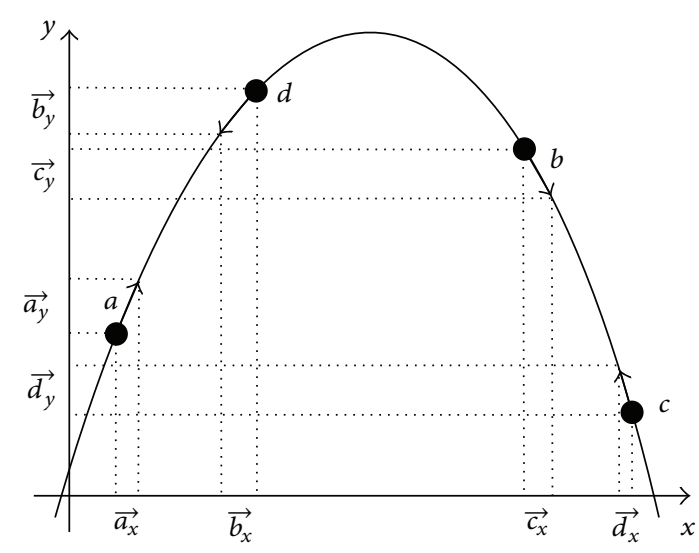

FIGURE 2: Basic principle of extremum seeking approach.

introduces the concept of PSO. Section 4 describes the system circuit architecture. Section 5 presents and discusses the experimental and numerical results. Finally, Section 6 provides some brief concluding remarks.

\section{ESC Technology and SMESC Controller Design}

2.1. Extremum Seeking Control (ESC) Scheme. Figure 1 presents a block diagram of a general ESC scheme. As shown, the major components comprise an integrator, a differentiator, a logic circuit, and an integral gain constant. In the present study, the aim of the ESC control scheme is to determine the global maximum value of the $x-y$ curve, (i.e., the $P$ - $V$ curve of the photovoltaic system).

According to Figure 1,

$$
\frac{d x}{d t}=K \varepsilon
$$

where $\varepsilon= \pm 1$ and $K$ is a positive constant. Furthermore,

$$
g=\frac{d y}{d t} .
$$

If $g<0$, then $\varepsilon=-1$. Conversely, if $g>0$, then $\varepsilon=1$.

Figure 2 shows the $d x / d t$ and $d y / d t$ signal changes corresponding to momenta $a, b, c$, and $d$, respectively [15]. Note that in the present study, $d x / d t$, and $d y / d t$ correspond to 


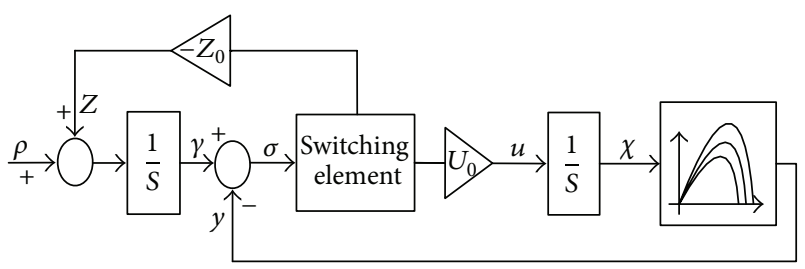

FIGURE 3: Block diagram of proposed SMESC scheme.

the rates of change of the voltage and the power, respectively. Since $(d y / d x)=(d y / d t) /(d x / d t)$, it follows that

$$
\begin{aligned}
\left.\left(\frac{d y}{d x}\right)\right|_{t=t^{-}} & >\left.0 \Longrightarrow\left(\frac{d x}{d t}\right)\right|_{t=t^{+}}=K, \\
\left.\left(\frac{d y}{d x}\right)\right|_{t=t^{-}} & <\left.0 \Longrightarrow\left(\frac{d x}{d t}\right)\right|_{t=t^{+}}=-K, \\
\frac{d x}{d t} & =K \cdot \operatorname{sign}\left(\frac{d y}{d x}\right)
\end{aligned}
$$

where sign() is a sign function.

According to (4), the balance point $d y / d x=0$ corresponds to the extremum of the $x-y$ curve in Figure 2. Moreover, system dynamics expressed in (4) depend on the slope of the $x-y$ curve. As shown in the following, the stability of the balance point can be confirmed by means of the Lyapunov theorem. Let the Lyapunov function be specified as follows:

$$
\bar{V}(t)=\frac{1}{2}\left(\frac{d y}{d x}\right)^{2} .
$$

Differentiating (5) with respect to time yields

$$
\dot{\bar{V}}(t)=\frac{d y}{d x} \frac{d^{2} y}{d x} \frac{d x}{d t}=\frac{d y}{d x} \frac{d^{2} y}{d x^{2}}\left(K \cdot \operatorname{sign}\left(\frac{d y}{d x}\right)\right) .
$$

According to Figure 2,

$$
\begin{gathered}
\frac{d^{2} y}{d x^{2}}<0, \\
\frac{d y}{d x} \operatorname{sign}\left(\frac{d y}{d x}\right)>0 .
\end{gathered}
$$

Therefore, selecting a positive value of $K$ yields $\bar{V}(t)<0$. Thus, the stability of the balance point is confirmed.

\subsection{Sliding Mode Extremum Seeking Control (SMESC)} Scheme. As shown in Figure 3, the robustness and stability of the ESC scheme used to solve the MPPT problem is enhanced in this study by adopting a sliding control law [16]. The stability of the resulting Sliding Mode ESC (SMESC) scheme is demonstrated in the following discussions.

In accordance with conventional control theory, the sliding surface in Figure 3 is defined as

$$
\sigma=\gamma-y .
$$

According to Figure 3,

$$
\begin{gathered}
u=\frac{d x}{d t}, \\
u=U_{0} \cdot \operatorname{sign}(\sigma), \\
\frac{d \gamma}{d t}=\rho+Z, \\
Z=-Z_{0} \cdot \operatorname{sign}(\sigma),
\end{gathered}
$$

where $Z_{0}$ and $U_{0}$ are both constants and $\rho$ is the maximum value of $y$ (power) versus $x$ (voltage). In photovoltaic system, $\rho$ can be obtained in advance after the irradiance was measured correctly. In this study, it is assumed that the irradiance can be measured correctly at any time.

Equation (9) can be replaced by

$$
\frac{d \sigma}{d t}=\rho-Z_{0} \cdot \operatorname{sign}(\sigma)-\frac{d y}{d x} \cdot U_{0} \cdot \operatorname{sign}(\sigma) .
$$

To ensure the stability of the MPPT system, $Z_{0}$ must be far larger than both $U_{0}$ and $\rho$, that is,

$$
\begin{gathered}
Z_{0} \gg U_{0}, \\
Z_{0} \gg \rho .
\end{gathered}
$$

To demonstrate the stability of the proposed SMESC scheme, let the Lyapunov function be defined as

$$
\bar{V}(t)=\frac{1}{2} \sigma^{2} .
$$

Differentiating (13) with respect to time $t$ yields

$$
\begin{aligned}
\dot{\bar{V}}(t) & =\sigma \dot{\sigma}=\sigma\left[\rho-Z_{0} \operatorname{sign}(\sigma)-U_{0} \frac{d y}{d x} \operatorname{sign}(\sigma)\right] \\
& =\sigma \rho-Z_{0}|\sigma|-U_{0} \frac{d y}{d x}|\sigma| \\
& \leq\left[\rho-Z_{0}-U_{0} \frac{d y}{d x}\right]|\sigma|,
\end{aligned}
$$

where $\rho$ is the maximum power of each solar panel in the photovoltaic array and is a given value, and $d y / d x$ approaches zero close to the maximum power point (MPP). Therefore, if $Z_{0} \gg U_{0}$ and $Z_{0} \gg \rho$, selecting appropriate values of $Z_{0}$ and $U_{0}$ yield the condition

$$
\dot{\bar{V}}(t)<0 .
$$

Thus, the stability of the proposed SMESC scheme is confirmed.

The ESC and SMESC schemes presented in Figures 1 and 3 , respectively, both use the sign function sign() to determine whether or not to change the signal. In theory, realizing an ideal switching function $\operatorname{sign}(\sigma)$ requires an infinitely high switching frequency. Such a switching frequency cannot be achieved in real-world systems. Thus, the switching function is generally achieved using ultrahigh speed switching 


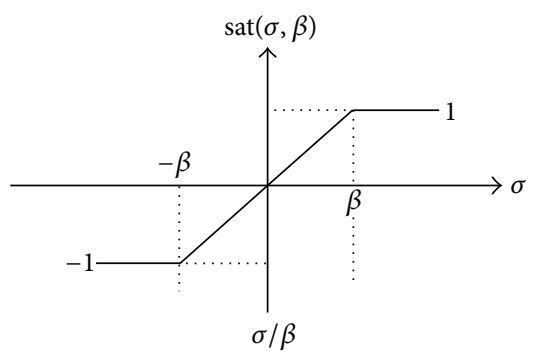

FIgURE 4: Sliding layer concept.

components instead. However, the continuous jumping of the system trajectory to the minimal space on either side of the sliding mode $(\sigma=0)$ results in high-frequency noise (chatter), which degrades the tracking performance of the MPPT controller and leads to energy losses. Amongst the various techniques available for suppressing the chattering phenomenon, the sliding layer concept is one of the simplest and most widely applied. As shown in Figure 4, the sliding layer control strategy replaces the $\operatorname{sign}(\sigma)$ function with the following function [17]:

$$
\begin{aligned}
\operatorname{sat}(\sigma, \beta) & = \begin{cases}1 ; & \sigma>\beta, \\
\frac{\sigma}{\beta} ; & |\sigma| \leq \beta, \\
-1 ; & \sigma<-\beta\end{cases} \\
& = \begin{cases}\operatorname{sign}(\sigma) ; & |\sigma|>\beta, \\
\frac{\sigma}{\beta} ; & |\sigma| \leq \beta .\end{cases}
\end{aligned}
$$

According to (8), the system space is divided into three regions, namely, $\sigma>\beta,|\sigma| \leq \beta$, and $\sigma<-\beta$, where $|\sigma| \leq$ $\beta$ represents the sliding layer. Furthermore, the robustness of the proposed SMESC controller is ensured by specifying $Z_{0} \gg \rho$, while the response time and stability are enhanced by determining the optimal value of the gain constant $\left(U_{0}\right)$ using a particle swarm optimization (PSO) algorithm. The details of the PSO algorithm are described in the following section.

\section{Particle Swarm Optimization (PSO) Algorithm}

PSO is a population-based stochastic optimization algorithm inspired by the social behavior of bird flocking or fish schooling. In the PSO optimization procedure, each candidate solution to the problem of interest is regarded as a volumeless particle (point) flying at a certain velocity in the $D$-dimensional search space. The velocity of each particle is adjusted dynamically according to its own flight experience and that of its companions. The position of the $i$ th particle is expressed as $x_{i}=\left(x_{i 1}, x_{i 2}, \ldots, x_{i D}\right)$, while the velocity of particle $i$ is expressed as $V_{i}=\left(V_{i 1}, V_{i 2}, \ldots, V_{i D}\right)$. The fitness (i.e., quality) of particle $i$ at each position is expressed as $P_{i}=\left(P_{i 1}, P_{i 2}, \ldots, P_{i D}\right)$. Furthermore, the best position of the particle so far, that is, the position which yields the greatest fit, is denoted as $P_{\text {best }}$. Among all of the particles, the instance

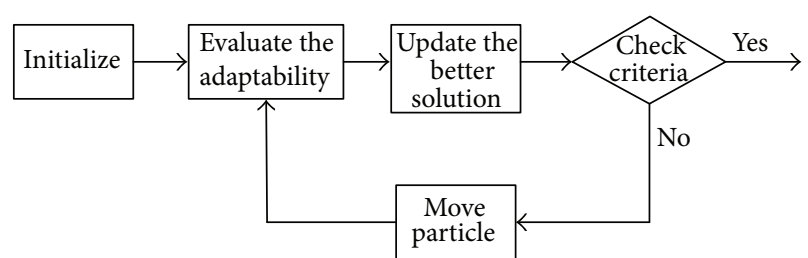

Figure 5: Flow chart of PSO search procedure.

of $P_{\text {best }}$ with the highest value is taken as the global optimal solution so far. During the iterative solution procedure, the velocity and position of each particle are updated as follows [18]:

$$
\begin{gathered}
V_{i d}=W \times V_{i d}+c_{1} \times \operatorname{Rand}() \times\left(P_{i d}-x_{i d}\right) \\
+c_{2} \times \operatorname{Rand}() \times\left(P_{\text {best }}-x_{i d}\right), \\
x_{i d}=x_{i d}+V_{i d},
\end{gathered}
$$

where $V_{i d}$ : velocity of particle $i$ in $d$ th dimension, $i$ : particle index, $d$ : dimension, $w$ : inertia weight, $c_{1}, c_{2}$ : learning constant, $\operatorname{Rand}()$ : random number between 0 and $1, P_{i d}$ : optimal position of particle $i$ up to now, $P_{\text {best }}$ : optimal position among all particle positions up to now, and $x_{i d}$ : current position of particle $i$ in $d$ th dimension.

Following the update process, the fitness of each particle is reevaluated. In the present study, the fitness evaluation is based on the following integrated absolute error (IAE) metric:

$$
\mathrm{IAE}=\int_{0}^{\infty}|\sigma| d \tau .
$$

The best position of each particle and the global best position among all of the particles are then updated if required. The particle update and evaluation procedure are repeated iteratively until the specified termination criteria are met. Having terminated the iteration process, the current global best position among all the particles is taken as the final optimal solution. Figure 5 presents a flow chart of the PSO procedure. The main steps in the PSO solution procedure can be summarized as follows [19]:

(1) specify the swarm size and initialize the position and velocity of each particle with random values,

(2) evaluate the fitness of each candidate solution $i$. If the fitness is better than the current best value, update $P_{\text {best }}$ accordingly,

(3) identify the best fitness so far among all the candidate solutions. If the fitness is better than the current best value, update it accordingly,

(4) if the termination criteria are met, end the search procedure,

(5) else, update the velocity and position of each particle using (17), respectively, and return to step (2). 


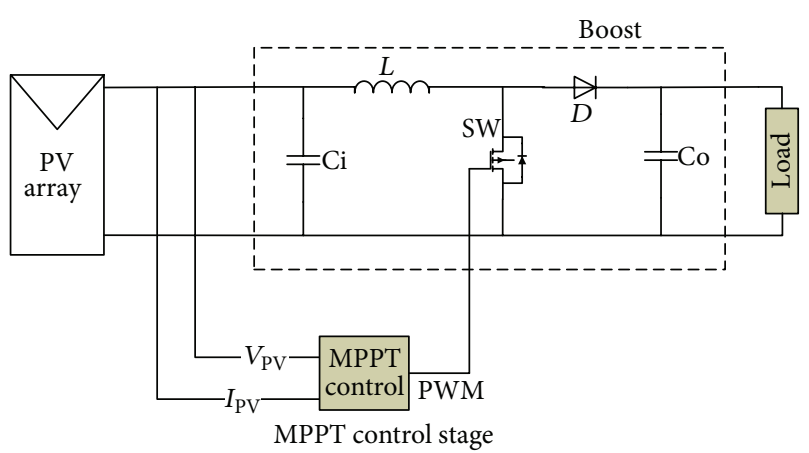

FIgURE 6: System structure.

TABLE 1: Specification of KYOCER KC30 solar panel.

\begin{tabular}{lcccc}
\hline$P_{\max }$ & $V_{\mathrm{MPP}}$ & $I_{\mathrm{MPP}}$ & $V_{\mathrm{OC}}$ & $I_{\mathrm{SC}}$ \\
\hline $30 \mathrm{~W}$ & $18 \mathrm{~V}$ & $1.71 \mathrm{~A}$ & $21.5 \mathrm{~V}$ & $1.8 \mathrm{~A}$ \\
\hline
\end{tabular}

Note that in the present study, the PSO search procedure is terminated when optimal value approaches a steady state.

\section{System Circuit Architecture}

Figure 6 illustrates the system structure adopted in the present study. As shown, the major components include a solar panel, a DC-DC boost converter, and the proposed SMESC controller. The details of each component are described in the following.

(1) Solar panel: maximum output power, $30 \mathrm{~W}$; maximum open-circuit voltage, $21.5 \mathrm{~V}$; maximum shortcircuit current, $1.8 \mathrm{~A}$; operating voltage at MPP, $18 \mathrm{~V}$; current at MPP, $1.71 \mathrm{~A}$.

(2) DC-DC boost converter: main circuit components include a transistor, a diode, an inductor, and a capacitor. The pulse width modulation (PWM) signal generated by the SMESC controller turns the transistor on and off as required, thereby completing the MPPT.

(3) MPPT controller: taking the voltage and current signals of the photovoltaic array as inputs, the SMESC controller calculates the PWM signal required to drive the DC-DC boost converter to switch the voltage in such a way as to approach the MPP. The controller is implemented using a dSPACE DS1104 $\mathrm{R} \& \mathrm{D}$ controller board which uses the computing technology of power PC as the core real-time computing system. The control strategy can be validated by actual test as soon as it is designed by using the computing capacity of DS1104 and the embedded inputoutput channel. The specifications are $250 \mathrm{MHz} \mathrm{CPU}$, 64-bit floating-point processor, $32 \mathrm{Mb}$ SDRAM global memory, $8 \mathrm{Mb}$ flash memory, 5 timer interrupts, 5 $\mathrm{ADC}$ channels, and $8 \mathrm{DAC}$ channels.

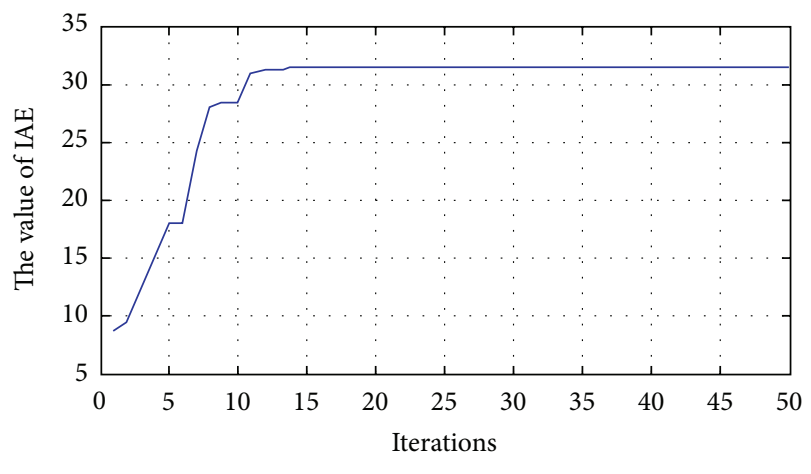

- IAE

FIGURE 7: Convergence characteristics of PSO algorithm.

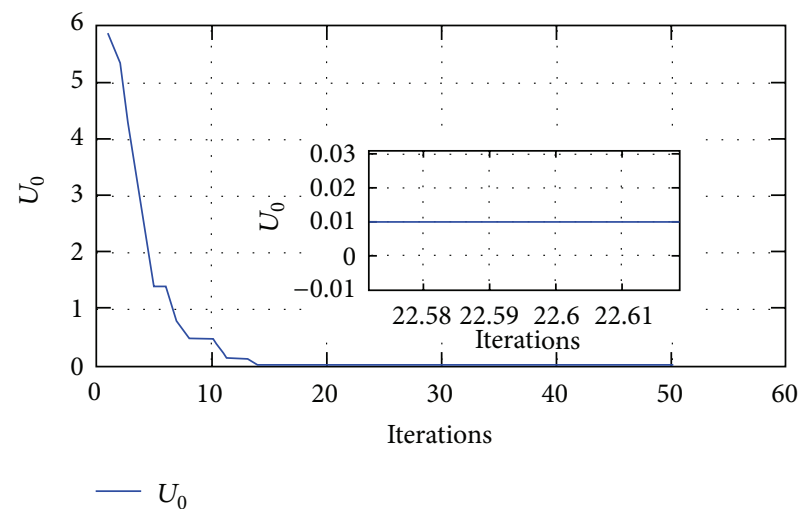

FIGURE 8: Conversion of $U_{0}$ during PSO solution procedure.

\section{Simulation and Experimental Results}

In this study, the performance of the proposed SMESC controller was evaluated both numerically and experimentally and was compared with that of two existing gradient detection-based MPPT algorithms, that is, $\mathrm{P} \& \mathrm{O}[20]$ and INC [21].

In both the simulations and the experimental investigation, the solar panel was a KYOCER KC30 panel with the specification shown in Table 1. As described in the previous section, the MPPT algorithm was implemented using a dSPACE R\&D control board. The numerical and experimental investigations considered two general irradiance conditions, namely (1) uniform irradiance $\left(1000 \mathrm{~W} / \mathrm{m}^{2}\right)$, and (2) nonuniform irradiance, that is, (i) a reduction from $1000 \mathrm{~W} / \mathrm{m}^{2}$ to $400 \mathrm{~W} / \mathrm{m}^{2}$ followed by a return to $1000 \mathrm{~W} / \mathrm{m}^{2}$ and (ii) an increase from $400 \mathrm{~W} / \mathrm{m}^{2}$ to $1000 \mathrm{~W} / \mathrm{m}^{2}$ followed by a return to $400 \mathrm{~W} / \mathrm{m}^{2}$.

5.1. Use of PSO Algorithm to Determine Optimal Value of Control Gain. As shown in Table 1, the solar panel has a maximum power of $30 \mathrm{~W}$ (i.e., $\rho=30$ ). As discussed in Section $2, Z_{0}$ should be far greater than $\rho$ in order to ensure the robustness and stability of the proposed SMESC scheme. Accordingly, in the present investigations, $Z_{0}$ was specified as 100. Meanwhile, the optimal value of the control gain $U_{0}$ 


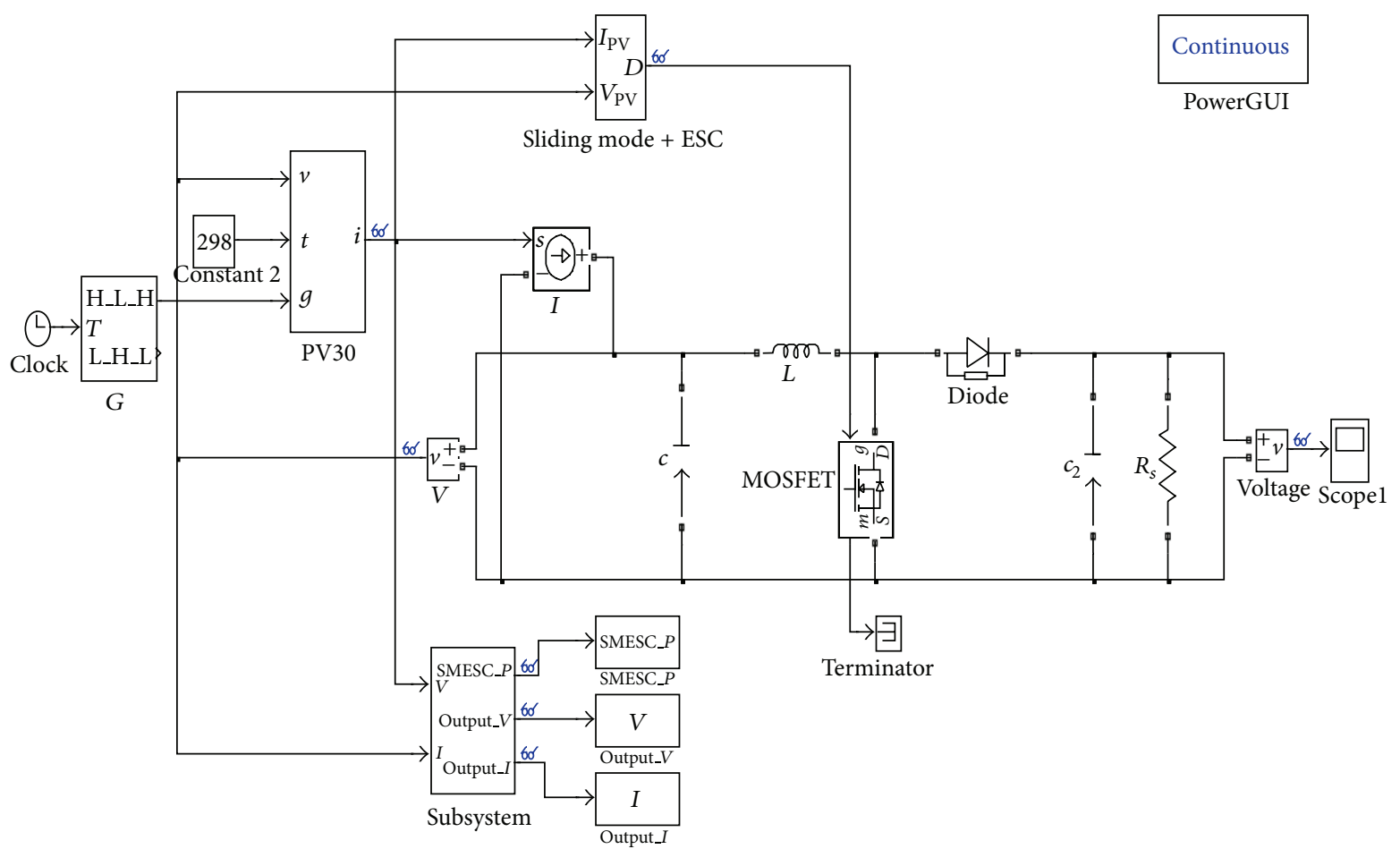

FIgURE 9: SMESC simulation model.

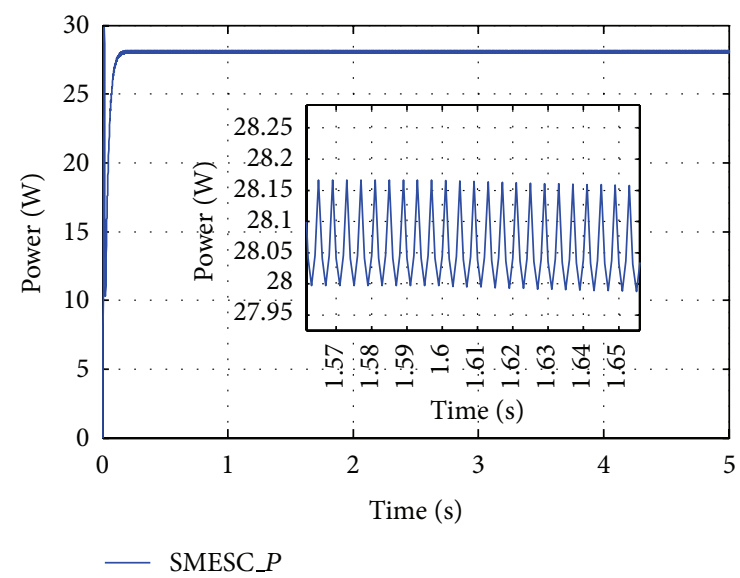

FIGURE 10: Simulation results for SMESC performance given uniform irradiance of $1000 \mathrm{~W} / \mathrm{m}^{2}$.

was obtained using the PSO optimization scheme. Figure 7 shows the convergence behavior of the PSO algorithm, while Figure 8 shows the convergence of $U_{0}$. It is seen in Figure 7 that the PSO algorithm converges after 15 iterations. From Figure 8, the optimal value of the control gain is equal to $U_{0}=0.01$. (Note that a gain of $U_{0}=0.01$ was therefore applied in all of the remaining simulations and experiments.)

5.2. Simulation Results. Figure 9 illustrates the simulation model adopted in the present study. Figure 10 presents the simulation results obtained for the output power using the proposed SMESC scheme given a uniform irradiance of

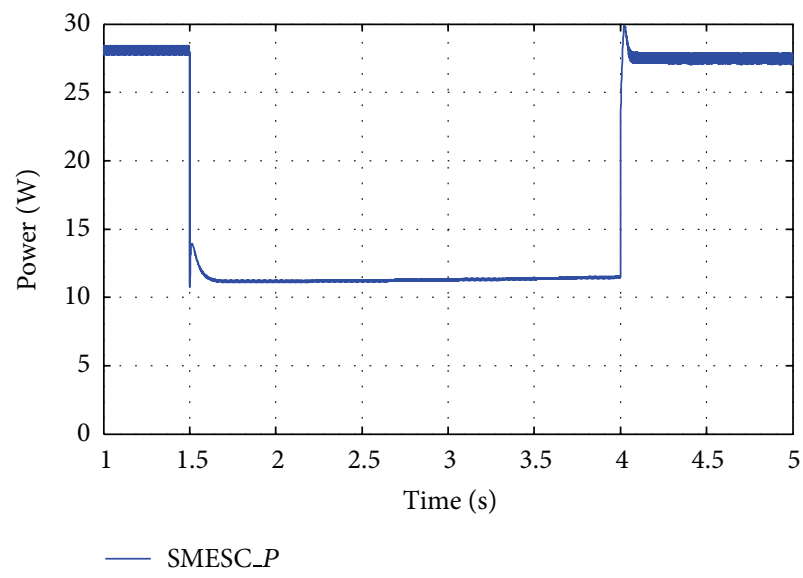

FIGURE 11: Simulation results for SMESC performance given step change in irradiance from $1000 \mathrm{~W} / \mathrm{m}^{2}$ to $400 \mathrm{~W} / \mathrm{m}^{2}$ followed by step change from $400 \mathrm{~W} / \mathrm{m}^{2}$ to $1000 \mathrm{~W} / \mathrm{m}^{2}$.

$1000 \mathrm{~W} / \mathrm{m}^{2}$. It is seen that the SMESC scheme enables a rapid and precise tracking of the MPP. Furthermore, it is observed that the proposed scheme yields a stable steadystate response.

To evaluate the robustness and ability of the SMESC scheme to track the MPP given dramatic variations in the irradiance conditions, a simulation was performed in which the irradiance was suddenly reduced from $1000 \mathrm{~W} / \mathrm{m}^{2}$ to $400 \mathrm{~W} / \mathrm{m}^{2}$ after $1.5 \mathrm{~s}$ and then restored to $1000 \mathrm{~W} / \mathrm{m}^{2}$ after an interval of $2.5 \mathrm{~s}$. The corresponding simulation results for the output power are shown in Figure 11. The results confirm 


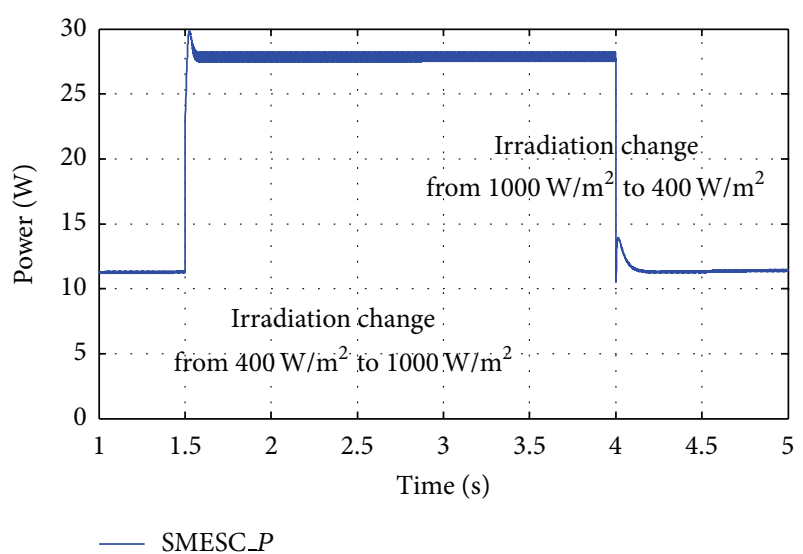

FIGURE 12: Simulation results for SMESC performance given step change in irradiance from $400 \mathrm{~W} / \mathrm{m}^{2}$ to $1000 \mathrm{~W} / \mathrm{m}^{2}$ followed by step change from $1000 \mathrm{~W} / \mathrm{m}^{2}$ to $400 \mathrm{~W} / \mathrm{m}^{2}$.

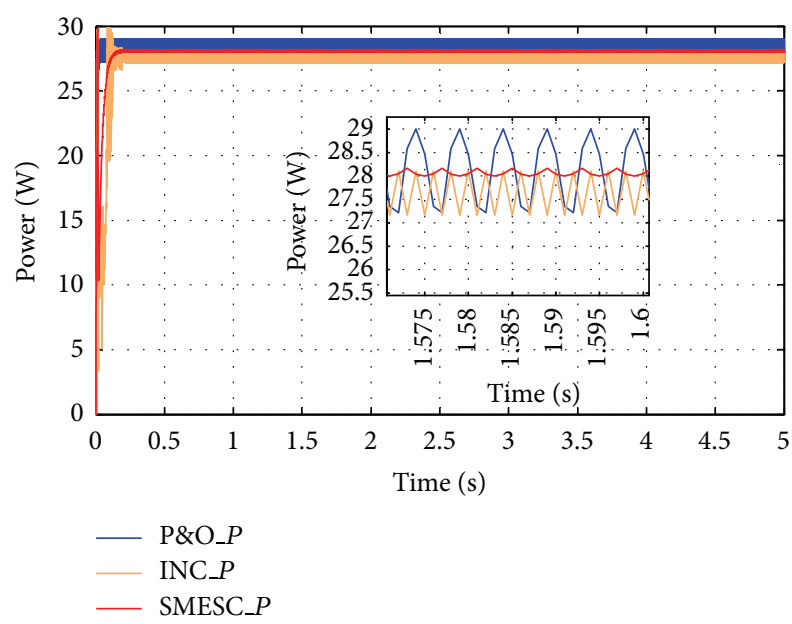

FIGURE 13: Simulation results for performance of P\&O, INC and SMESC schemes given uniform irradiance of $1000 \mathrm{~W} / \mathrm{m}^{2}$.

that the SMESC controller rapidly tracks the MPP as the irradiance is first reduced and then increased. Figure 12 shows the simulated output power for the case where the irradiance is increased abruptly from $400 \mathrm{~W} / \mathrm{m}^{2}$ to $1000 \mathrm{~W} / \mathrm{m}^{2}$ after $1.5 \mathrm{~s}$ and then reduced to $400 \mathrm{~W} / \mathrm{m}^{2}$ after a further $2.5 \mathrm{~s}$. The results again confirm the robustness and ability of the SMESC scheme to rapidly track the MPP given a dramatic variation in the irradiance intensity.

Figure 13 compares the MPPT performance of the $\mathrm{P} \& \mathrm{O}$, INC, and SMESC schemes given a uniform irradiance of $1000 \mathrm{~W} / \mathrm{m}^{2}$. As shown, the $\mathrm{P} \& \mathrm{O}$ scheme results in violent fluctuations in the output power under steady-state conditions and therefore leads to a significant energy loss. It is seen that the INC scheme results in an improved steady-state performance. However, fluctuations in the output power are still observed. Furthermore, the tracking performance of INC is highly sensitive to the accuracy of the signal measurements and is therefore liable to significant error in real-world

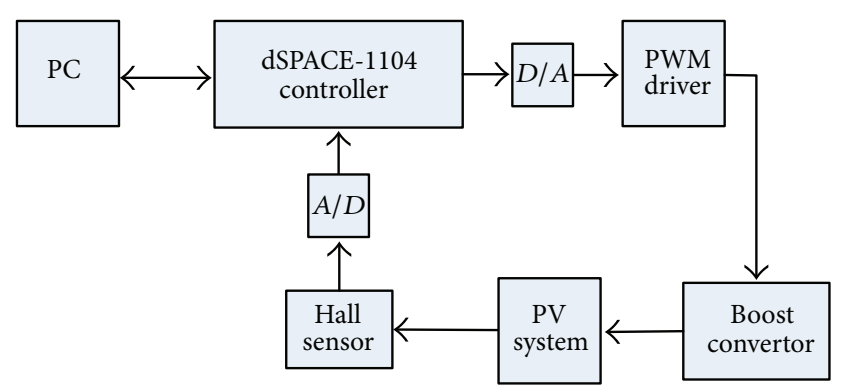

FIGURE 14: Hardware architecture used to evaluate real-world performance of SMESC controller.

implementations. Notably, the $\mathrm{P} \& \mathrm{O}$ and INC methods both use a gradient detection-based approach to identify the system signals. The resulting ultra-high switching frequency not only leads to energy loss but also increases the risk of component damage. It is seen that the proposed SMESC scheme results in a far better steady-state performance than the $\mathrm{P} \& \mathrm{O}$ or INC scheme. In other words, the results confirm the effectiveness of the sliding layer control scheme in suppressing the chattering phenomenon and reducing power loss as a result.

5.3. Experimental Results. Having demonstrated the basic validity of the proposed SMESC controller via MATLAB simulations, a series of experimental investigations was performed using the hardware architecture shown in Figure 14 to evaluate the real-world applicability of the proposed scheme.

Figure 15 presents a block diagram showing the implementation of the SMESC scheme using the dSPACE control board. Note that "Filter_V" and "Filter_I" are wave filters, "Sliding mode" is the sliding controller, "SMESC" is the proposed Sliding Mode extremum seeking control scheme, and "PWM" is the PWM circuit.

Figure 16 shows the experimental results obtained for the output power given a uniform irradiance of $1000 \mathrm{~W} / \mathrm{m}^{2}$. The results confirm the ability of the proposed control scheme to ensure the system stability and efficiency under steady-state conditions. In addition, it is observed that the SMESC controller responds rapidly to the step change in the irradiance, thereby further reducing energy losses during the MPPT procedure.

Figure 17 shows the experimental results for the output power waveform given a sudden reduction of the irradiance from $1000 \mathrm{~W} / \mathrm{m}^{2}$ to $400 \mathrm{~W} / \mathrm{m}^{2}$ followed by an abrupt return to an irradiance of $1000 \mathrm{~W} / \mathrm{m}^{2}$ after an interval of $2.5 \mathrm{~s}$. It is seen that the SMESC controller accurately and rapidly tracks the MPP as the irradiance is first reduced and then increased. Figure 18 presents the experimental output power waveform for the case in which the irradiance is first increased from $400 \mathrm{~W} / \mathrm{m}^{2}$ to $1000 \mathrm{~W} / \mathrm{m}^{2}$ and then restored to $400 \mathrm{~W} / \mathrm{m}^{2}$. The results again confirm that the proposed scheme yields both a rapid transient response and a stable steady-state response.

Figure 19 compares the experimental output power waveforms obtained using the $\mathrm{P} \& \mathrm{O}$, INC, and SMESC control schemes, respectively, given a uniform irradiance of 


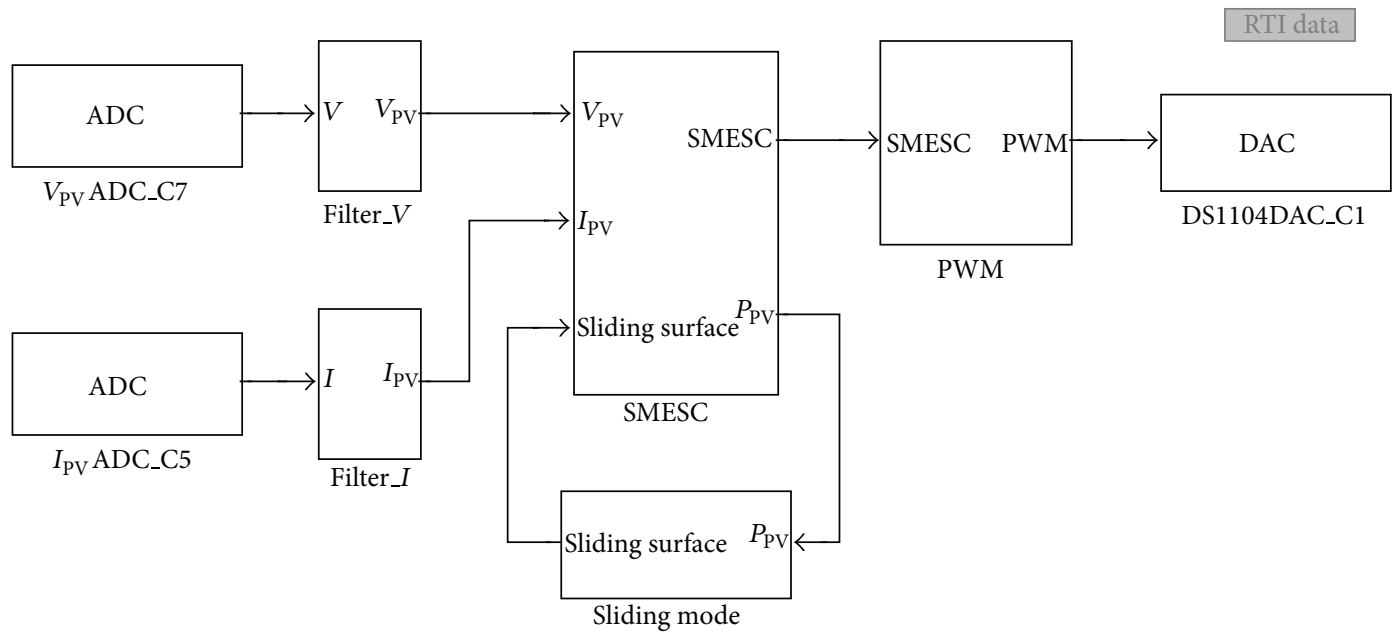

FIGURE 15: SMESC processing block.

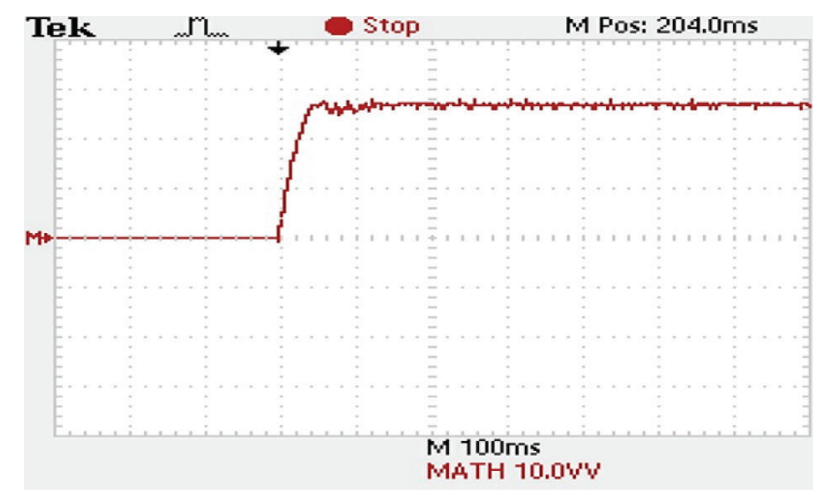

FIGURE 16: Experimental results for SMESC performance given uniform irradiance of $1000 \mathrm{~W} / \mathrm{m}^{2}$.

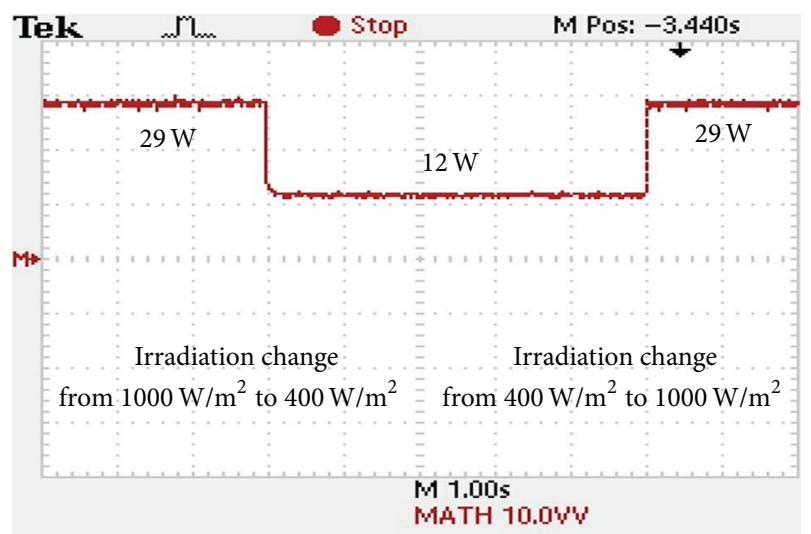

FIGURE 17: Experimental results for SMESC performance given step change in irradiance from $1000 \mathrm{~W} / \mathrm{m}^{2}$ to $400 \mathrm{~W} / \mathrm{m}^{2}$ followed by step change from $400 \mathrm{~W} / \mathrm{m}^{2}$ to $1000 \mathrm{~W} / \mathrm{m}^{2}$.

$1000 \mathrm{~W} / \mathrm{m}^{2}$. The results show that the P\&O scheme leads to significant oscillations in the output power under steadystate conditions. Thus, while the $\mathrm{P} \& \mathrm{O}$ scheme has a simple architecture and is easily implemented, it has poor efficiency

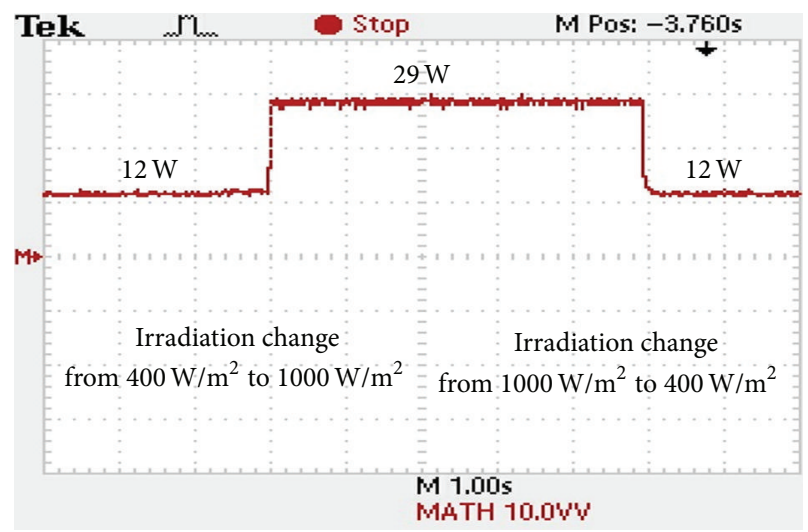

FIGURE 18: Experimental results for SMESC performance given step change in irradiance from $400 \mathrm{~W} / \mathrm{m}^{2}$ to $1000 \mathrm{~W} / \mathrm{m}^{2}$ followed by step change from $1000 \mathrm{~W} / \mathrm{m}^{2}$ to $400 \mathrm{~W} / \mathrm{m}^{2}$.

and results in significant power losses. It is seen that the INC scheme yields a lower steady-state oscillation of the output power waveform than the $\mathrm{P} \& \mathrm{O}$ method. However, the tracking accuracy and efficiency of INC are both highly sensitive to the accuracy of the sensors used to acquire the system signal. The results presented in Figure 19 confirm that the proposed SMESC scheme successfully resolves the chattering phenomenon associated with traditional gradient detection-based methods and yields an accurate and highly efficient MPP tracking performance.

\section{Conclusions}

This study has proposed a sliding mode extremum seeking control (SMESC) scheme for maximum power point (MPP) tracking in photovoltaic power generation systems. In the proposed approach, the chattering phenomenon associated with the ultrahigh switching frequency required in traditional gradient-detection methods is suppressed by means of a sliding layer concept. Moreover, the response time and 


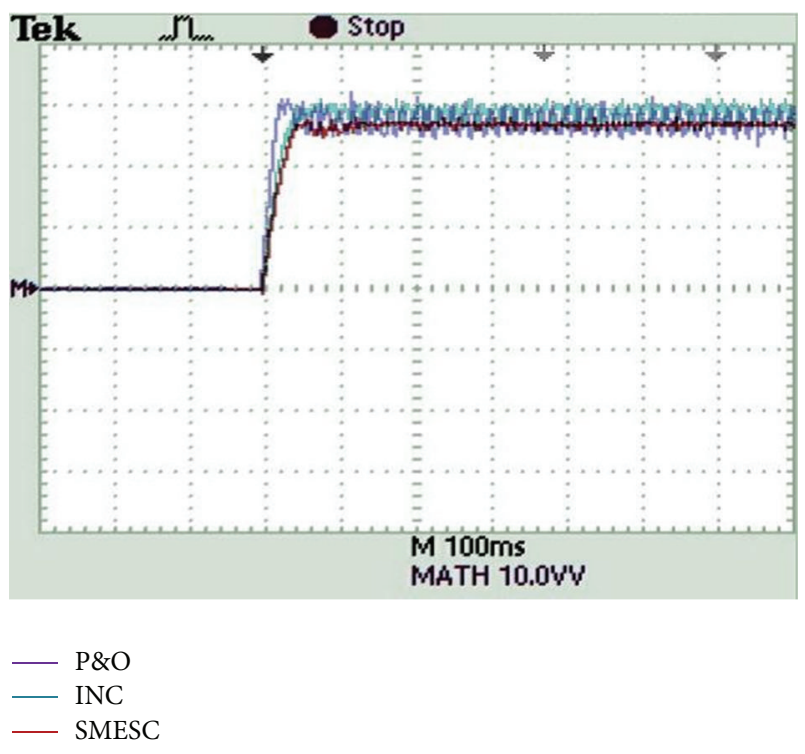

Figure 19: Comparison of experimental results obtained using $\mathrm{P} \& \mathrm{O}, \mathrm{INC}$, and SMESC schemes.

stability of the proposed control scheme are enhanced by using a particle swarm optimization algorithm to determine the optimal value of the control gain. The validity of the proposed scheme has been investigated numerically and experimentally under both uniform irradiance conditions and rapidly varying irradiance conditions. The results have shown that the proposed scheme provides a rapid and highly accurate MPP tracking performance. In addition, it has been shown that the scheme minimizes oscillations in the output power under steady-state conditions and therefore results in a better efficiency than traditional MPPT algorithms such as $\mathrm{P} \& \mathrm{O}[20]$ and INC [21].

\section{Acknowledgment}

The authors would like to thank the National Science Council, Taiwan, for financially supporting this research under Contract no. NSC 100-2221-E-027-015 and NSC-2628-E-167-002MY3.

\section{References}

[1] K. Ishaque, Z. Salam, M. Amjad, and S. Mekhilef, "An improved particle swarm optimization (PSO)-based MPPT for PV with reduced steady-state oscillation," IEEE Transactions on Power Electronics, vol. 27, no. 8, pp. 3627-3638, 2012.

[2] M. Kritiker and A. Goykadosh, "Renewable and sustainable energy replacement sources," in Proceedings of the Long Island Systems, Applications and Technology Conference (LISAT '10), pp. 1-4, May 2010.

[3] N. Femia, G. Petrone, G. Spagnuolo, and M. Vitelli, "Optimizing duty-cycle perturbation of P\&O MPPT technique," in Proceedings of the 35th IEEE Annual Power Electronics Specialists Conference (PESC '04), pp. 1939-1944, June 2004.
[4] A. K. Abdelsalam, A. M. Massoud, S. Ahmed, and P. N. Enjeti, "High-performance adaptive Perturb and observe MPPT technique for photovoltaic-based microgrids," IEEE Transactions on Power Electronics, vol. 26, no. 4, pp. 1010-1021, 2011.

[5] J. Li and H. Wang, "A novel stand-alone PV generation system based on variable step size INC MPPT and SVPWM control," in Proceedings of the 6th IEEE International Power Electronics and Motion Control Conference (IPEMC '09), pp. 2155-2160, May 2009.

[6] A. Safari and S. Mekhilef, "Simulation and hardware implementation of incremental conductance MPPT with direct control method using cuk converter," IEEE Transactions on Industrial Electronics, vol. 58, no. 4, pp. 1154-1161, 2011.

[7] F. Liu, S. Duan, F. Liu, B. Liu, and Y. Kang, "A variable step size INC MPPT method for PV systems," IEEE Transactions on Industrial Electronics, vol. 55, no. 7, pp. 2622-2628, 2008.

[8] E. Koutroulis, K. Kalaitzakis, and N. C. Voulgaris, "Development of a microcontroller-based, photovoltaic maximum power point tracking control system," IEEE Transactions on Power Electronics, vol. 16, no. 1, pp. 46-54, 2001.

[9] A. M. Bazzi and P. T. Krein, "Concerning maximum power point tracking for photovoltaic optimization using ripplebased extremum seeking control," IEEE Transactions on Power Electronics, vol. 26, no. 6, pp. 1611-1612, 2011.

[10] H. T. Yau and C. H. Wu, "Comparison of extremum-seeking control techniques for maximum power point tracking in photovoltaic systems," Energies, vol. 4, pp. 2180-2195, 2011.

[11] K. Ariyur and M. Krstic, Real-Time Optimization by Extremum Seeking Control, Wiley, New York, NY, USA, 2003.

[12] K. B. Ariyur and M. Krstić, "Analysis and design of multivariable extremum seeking," in Proceedings of the American Control Conference, vol. 4, pp. 2903-2908, May 2002.

[13] S. L. Brunton, C. W. Rowley, S. R. Kulkarni, and C. Clarkson, "Maximum power point tracking for photovoltaic optimization using ripple-based extremum seeking control," IEEE Transactions on Power Electronics, vol. 25, no. 10, pp. 2531-2540, 2010.

[14] C. C. Chu and C. L. Chen, "Robust maximum power point tracking method for photovoltaic cells: a sliding mode control approach," Solar Energy, vol. 83, no. 8, pp. 1370-1378, 2009.

[15] R. Leyva, C. Alonso, I. Queinnec, A. Cid-Pastor, D. Lagrange, and L. Martínez-Salamero, "MPPT of photovoltaic systems using extremum-seeking control," IEEE Transactions on Aerospace and Electronic Systems, vol. 42, no. 1, pp. 249-258, 2006.

[16] C. Olalla, M. I. Arteaga, R. Leyva, and A. El Aroudi, "Analysis and comparison of extremum seeking control techniques," in Proceedings of IEEE International Symposium on Industrial Electronics (ISIE '07), pp. 72-76, June 2007.

[17] J. J. Slotine and S. S. Sastry, "Tracking control of non-linear systems using sliding surfaces, with application to robot manipulators," International Journal of Control, vol. 38, no. 2, pp. 465492, 1983.

[18] B. Liu, L. Wang, and Y. H. Jin, "An effective PSO-based memetic algorithm for flow shop scheduling," IEEE Transactions on Systems, Man, and Cybernetics, Part B, vol. 37, no. 1, pp. 18-27, 2007.

[19] X. Chen and Y. Li, "A modified PSO structure resulting in high exploration ability with convergence guaranteed," IEEE Transactions on Systems, Man, and Cybernetics, Part B, vol. 37, no. 5, pp. 1271-1289, 2007. 
[20] M. A. Elgendy, B. Zahawi, and D. J. Atkinson, "Assessment of perturb and observe MPPT algorithm implementation techniques for PV pumping applications," IEEE Transactions on Sustainable Energy, vol. 3, no. 1, pp. 21-33, 2012.

[21] Q. Mei, M. Shan, L. Liu, and J. M. Guerrero, "A novel improved variable step-size incremental-resistance MPPT method for PV systems," IEEE Transactions on Industrial Electronics, vol. 58, no. 6, pp. 2427-2434, 2011. 

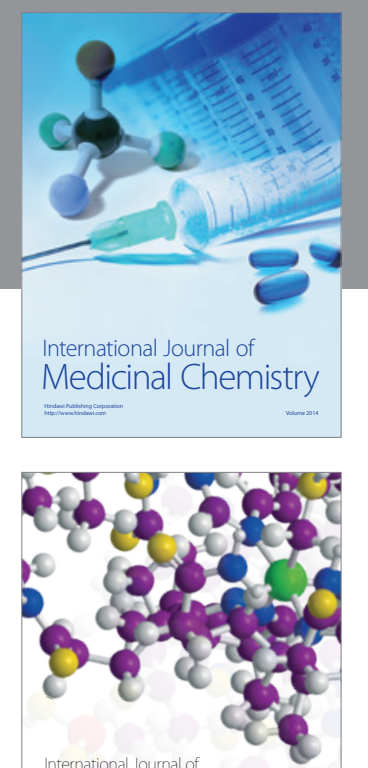

\section{Carbohydrate} Chemistry

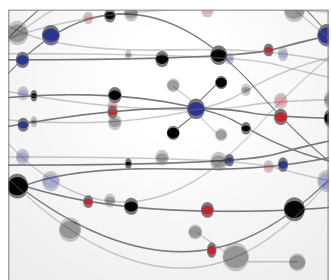

The Scientific World Journal
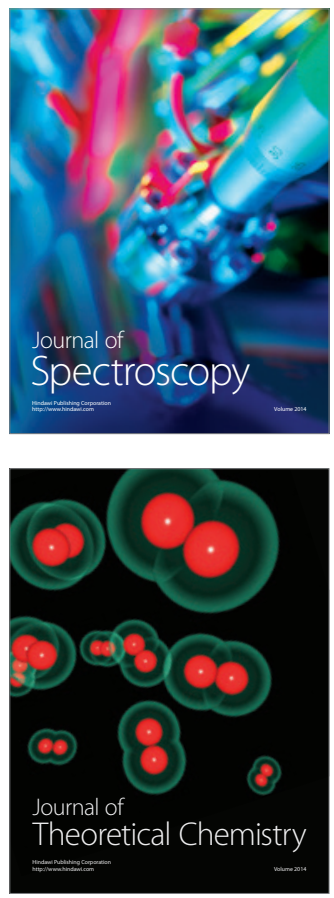
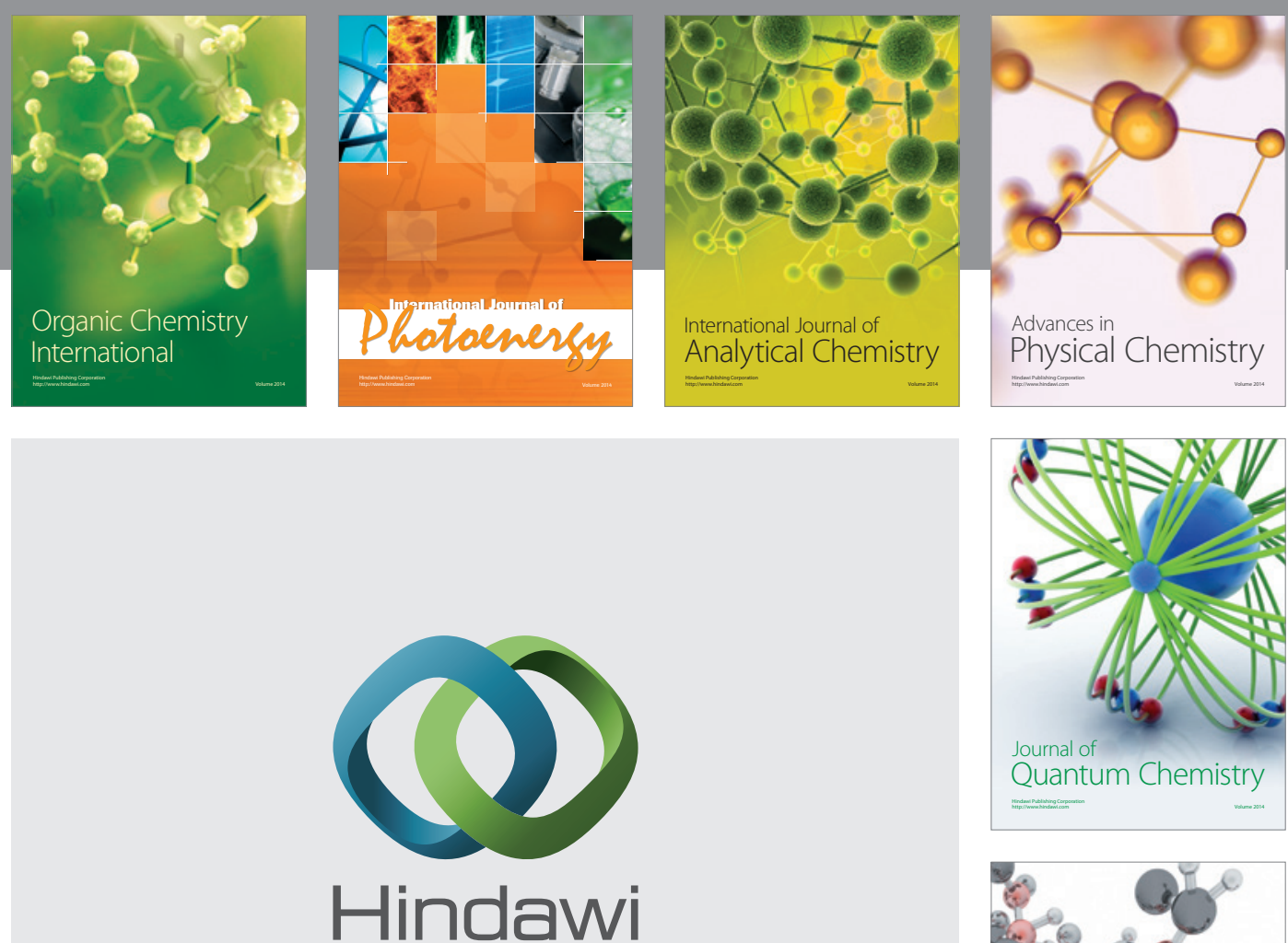

Submit your manuscripts at

http://www.hindawi.com

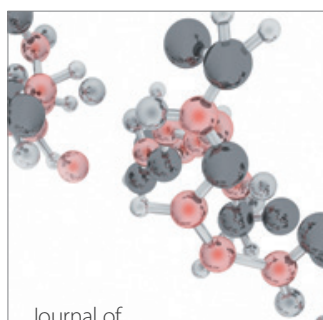

Analytical Methods

in Chemistry

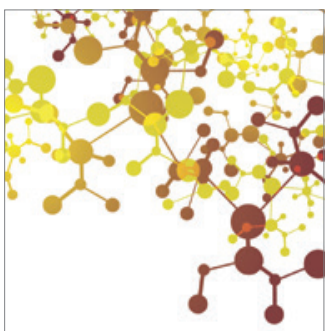

Journal of

Applied Chemistry

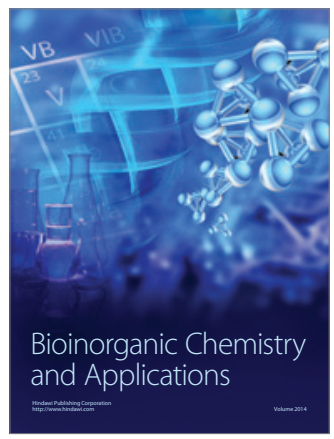

Inorganic Chemistry
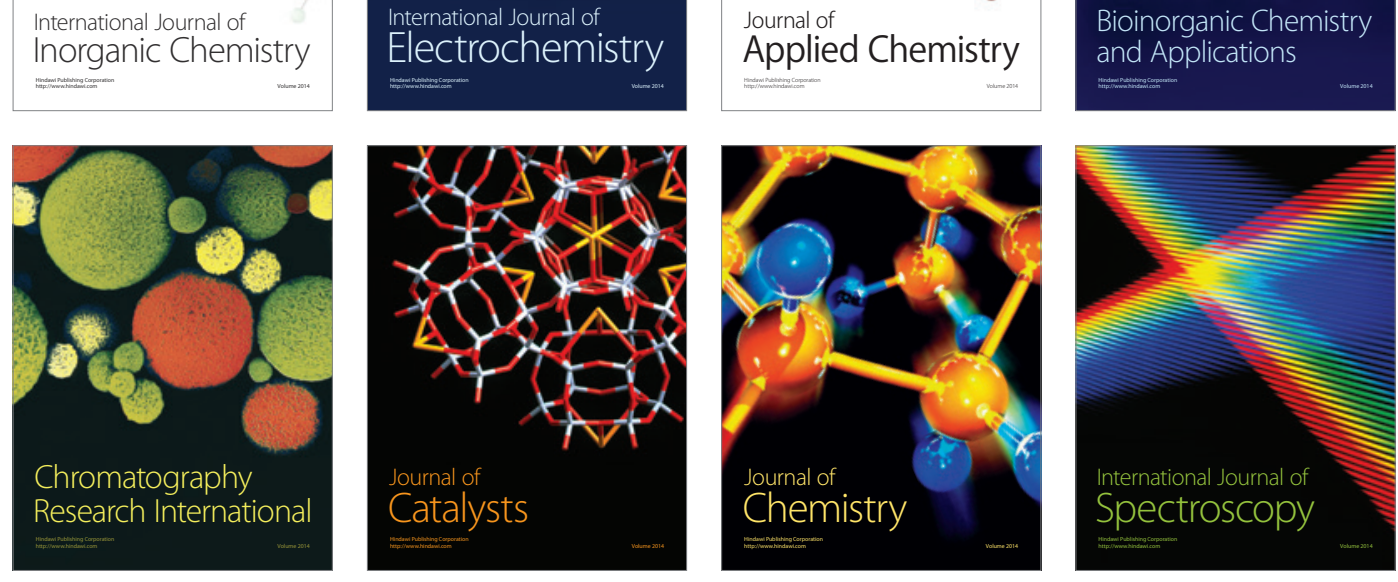